\title{
Estimation of soil properties using observations and the crop model STICS. Interest of global sensitivity analysis and impact on the prediction of agro-environmental variables
}

\author{
Hubert Varella, Martine Guérif and Samuel Buis \\ INRA-UAPV EMMAH
}

France

\section{Introduction}

Dynamic crop models are very useful to predict the behaviour of crops in their environment and are widely used in a lot of agro-environmental work such as crop monitoring, yield prediction or decision making for cultural practices (Batchelor et al., 2002; Gabrielle et al., 2002; Houlès et al., 2004). These models usually have many parameters and their estimation is a major problem for agro-environmental prediction (Tremblay and Wallach, 2004; Makowski et al., 2006). For spatial application, the knowledge of soil parameters is crucial since they are responsible for a major part of the variability of the crop model output variables of interest (Irmak et al., 2001; Launay and Guérif, 2003; Ferreyra et al., 2006). These parameters may be estimated from different techniques: either with soil analysis at the different points of the study area, from a soil map and the application of pedotransfer functions (Reynolds et al., 2000; Murphy et al., 2003), from remote sensing images (Lagacherie et al., 2008) or by using electrical resistivity measurements (Golovko and Pozdnyakov, 2007). The choice of the first method is difficult because of practical limitations, as well as time and financial constraints. Detailed soil maps adapted to the scale of precision agriculture and even to the scale of catchment are scarcely available (King et al., 1994), while the use of remote sensing images or electrical resistivity is still hampered by a lack of robust interpretation of the signal (Lagacherie et al., 2008). Moreover, these techniques do not permit to access the values of all the soil parameters required to apply a complex crop model. Fortunately, techniques derived from remote sensing images (Weiss and Baret, 1999; Houborg and Boegh, 2008) or yield monitoring (Blackmore and Moore, 1999; Pierce et al., 1999) allow soil parameters being estimated through the inversion of crop models.

Estimating parameters of complex models such as crop models may be not so easy (Tremblay and Wallach, 2004; Launay and Guérif, 2005). One of the reasons for the difficulties encountered may be a lack of sensitivity of the observed variables to the parameters, making the estimation process inefficient. Another reason may be that the influence of the parameters on the observed variables takes place mainly through 
interactions, making it difficult to identify the relevant factors (Saltelli et al., 2000). For complex non-linear models such as crop models, global sensitivity analysis (GSA) methods are able to give relevant information on the sensitivity of model outputs to the whole range of variation of model inputs. Many studies have focused on this subject, namely, how to choose the main parameters to be estimated for the model calibration (Campolongo and Saltelli, 1997; Ruget et al., 2002; Gomez-Delgado and Tarantola, 2006; Makowski et al., 2006) and ranked the importance of the parameters by calculating global sensitivity indices: first-order indices (the main effect of the parameter on the output) and total indices (sum of all effects involving the parameter, including the interactions with other parameters). The common practice is consistent with the principles expressed by Ratto et al. (2007). Small total sensitivity indices indicate a negligible effect of the parameter on the model output concerned. These parameters can be fixed at a nominal value ("Factor Fixing setting"). High first-order indices reveal a clearly identifiable influence of the parameter on the model output concerned, and therefore the parameters need to be determined accurately ("Factor Prioritization setting"). Small first-order indices combined with large interaction indices result in a lack of identification. In practice, the two first rules are commonly used to select the set of parameters to be estimated in a calibration problem. GSA can also be used to evaluate the quantity of information contained in a given set of observations for estimating parameters and thus to determine which is the best observation set for estimating the parameters (Kontoravdi et al., 2005). Although the results of GSA are often used to design the estimation process, the link between GSA indices and the quality of parameter estimation has never been quantified.

Our objectives in this study are twofold. Firstly, we propose to use GSA results in order to measure the quantity of information contained in different sets of observations and to illustrate the link between this measurement and the quality of parameter estimates. Secondly, we propose to study the impact of the quality of parameter estimates on the prediction of variables of interest for agro-environmental work. As the performance of the estimation process is supposed to depend on several conditions such as soil type, cropping conditions (preceding crop and climate) or available observations, we chose to conduct the study on synthetic observations in order to be able to generate variability in parameter retrieval performance as well as in sensitivity structure of the observed model outputs to soil parameters and in the prediction performance. This choice also allows eliminating the impact of model errors, which may complicate the interpretation of the results. Finally, we considered in this study the STICS-wheat crop model and various synthetic observations on wheat crops: derived from remote sensing images (LAI and absorbed nitrogen) as well as grain yield.

\section{Material and methods}

\subsection{The crop model, output variables and soil parameters}

\subsubsection{The STICS model}

The STICS model (Brisson et al., 2002) is a nonlinear dynamic crop model simulating the growth of various crops. For a given crop, STICS takes into account the climate, type of soil and cropping techniques to simulate the carbon, water and nitrogen balances of the crop-soil system on a daily time scale. In this study, a wheat crop is simulated. The crop is 
essentially characterized by its above-ground biomass carbon and nitrogen, and leaf area index. The main outputs are agronomic variables (yield, grain protein content) as well as environmental variables (water and nitrate leaching). Yield, grain protein content and nitrogen balance in the soil at harvest are of particular interest for decision making, especially for monitoring nitrogen fertilization (Houlès et al., 2004). Nitrogen absorbed by the plant and leaf area index are also important to analyze the health and growth of the plant during the crop's growing season.

The STICS model includes more than 200 parameters arranged in three main groups: those related to the soil, those related to the characteristics of the plant or to the genotype, and those describing the cropping techniques. The values of the last group of parameters are usually known as they correspond to the farmer's decisions. The parameters related to the plant are generally determined either from literature, from experiments conducted on specific processes included in the model (e.g. mineralization rate, critical nitrogen dilution curve etc.) or from calibrations based on large experimental database, as is the case for the STICS model (Hadria et al., 2007). The soil parameters are difficult to determine at each point of interest and are responsible for a large part of the spatial variability of the output variable. That is why the sensitivity analysis and parameter estimation processes described in this study only concern soil parameters.

\subsubsection{Output variables considered}

In this study, we focus on two types of STICS output variables. First, those corresponding with observations that may be done on wheat canopy by automated measurements. They consist in:

- the leaf area index $\left(L A I_{t}\right)$ and the nitrogen absorbed by the plant $\left(Q N_{t}\right)$ at various dates $t$ during the crop season - as potentially derived from remote sensing image inversion (Weiss and Baret, 1999; Houborg and Boegh, 2008),

- the yield at harvest $(Y l d)$ as potentially provided by yield monitoring systems.

These output variables, hereafter referred to as "observable variables" can be observed at different dates during the growing season.

Second, a main objective of this study, beyond the estimation of soil parameter values, lies in the prediction of some output variables of interest, and its improvement as compared to the prediction obtained with a lack of precise values on soil parameters. They consist in:

- yield at harvest (Yld),

- protein in the grain at harvest (Prot),

- nitrogen contain in the soil at harvest (Nit).

Yield, grain protein content and nitrogen balance in the soil at harvest are of particular interest for decision making, especially for monitoring nitrogen fertilization (Houlès et al., 2004). Nitrogen absorbed by the plant are also important to analyze the health and growth of the plant during the crop's growing season (Baret et al., 2006).

\subsubsection{The soil parameters estimated}

The STICS model contains about 60 soil parameters. In our case, in order to limit the problems of identifiability, the number of soil parameters to be estimated has been reduced. First, among the available options for simulating the soil system, the simplest was chosen, by ignoring capillary rise and nitrification. These assumptions define the 
domain of validity of the model considered and hence, of the results that are found. We then considered the soil as a succession of two horizontal layers, each characterized by a specific thickness parameter. From the observation of the tillage practices in the region around our experimental site of Chambry $\left(49.35^{\circ} \mathrm{N}, 3.37^{\circ} \mathrm{E}\right)$ (Guérif et al., 2001), the thickness of the first layer was set at $0.30 \mathrm{~m}$. We performed a first sensitivity analysis on the 13 resulting soil parameters. This allowed us to fix those whose effects on the observed variables were negligible: for each parameter we computed the values of its effects on all the observed variables considered for a lot of soil, climate and agronomic conditions, and dropped the parameters for which all these values were less than $10 \%$ of the total effects generated by the 13 parameters. We thus restricted the study to 7 parameters.

The 7 soil parameters considered (Table 1) characterize both water and nitrogen processes. They refer to permanent characteristics and initial conditions. Among the permanent characteristics, clay and organic nitrogen content of the top layer are involved mainly in organic matter decomposition processes and nitrogen cycle in the soil. Water content at field capacity of both layers affects the water (and nitrogen) movements and storage in the soil reservoir. Finally, the thickness of the second layer defines the volume of the reservoir. The initial conditions correspond to the water and nitrogen content, Hinit and NO3init, at the beginning of the simulation, in this case the sowing date.

\begin{tabular}{|c|c|c|c|}
\hline Parameter & Definition & Range & Unit \\
\hline $\operatorname{argi}$ & Clay content of the 1rst layer & $14-37$ & $\%$ \\
\hline Norg & Organic nitrogen content of the 1 rst layer & $0.049-0.131$ & $\%$ \\
\hline $\operatorname{epc}(2)$ & Thickness of the 2nd layer & $0-70$ or $50-130^{*}$ & $\mathrm{~cm}$ \\
\hline HCC (1) & Water content at field capacity (1rst layer) & $14-30$ & g g-1 \\
\hline HCC (2) & Water content at field capacity ( 2 nd layer) & $14-30$ & g g-1 \\
\hline Hinit & Initial water content (both layers) & $4-29$ & $\%$ of weight \\
\hline NO3init & Initial mineral nitrogen content (1rst layer) & $4-21.5$ or $25-86^{* *}$ & kg N ha-1 \\
\hline
\end{tabular}

\subsection{Global sensitivity analysis}

In this study, we chose a variance-based method of global sensitivity analysis which allows calculating the sensitivity indices for a non-linear model such as STICS. More precisely, the method we chose is Extended FAST.

\subsubsection{Variance decomposition and sensitivity indices}

We denote a given output variable of the STICS model as $Y$. The total variance of $Y$, $V(Y)$, caused by variation in the 7 selected soil parameters $\theta$, can be partitioned as follows (Chan et al., 2000):

$$
V(Y)=\sum_{i=1}^{7} V_{i}+\sum_{1 \leq i<j \leq 7} V_{i j}+\cdots+V_{1,2, \cdots, 7}
$$


where $V_{i}=V\left[E\left(Y \mid \theta_{\mathrm{i}}\right)\right]$ measures the main effect of the parameter $\theta_{i}, i=1, \cdots, 7$, and the other terms measure the interaction effects. Decomposition (2) is used to derive two types of sensitivity indices defined by:

$$
\begin{gathered}
S_{i}=\frac{V_{i}}{V(Y)} \\
S T_{i}=\frac{V(Y)-V_{-i}}{V(Y)}
\end{gathered}
$$

where $V_{-i}$ is the sum of all the variance terms that do not include the index $i$.

$S_{i}$ is the first-order (or main) sensitivity index for the $i^{\text {th }}$ parameter. It computes the fraction of $Y$ variance explained by the uncertainty of parameter $\theta_{i}$ and represents the main effect of this parameter on the output variable $Y$.

$S T_{i}$ is the total sensitivity index for the $i^{\text {th }}$ parameter and is the sum of all effects (first and higher order) involving the parameter $\theta_{i}$.

$S_{i}$ and $S T_{i}$ are both in the range $(0,1)$, low values indicating negligible effects, and values close to 1 huge effects. $S T_{i}$ takes into account both $S_{i}$ and the interactions between the $i^{\text {th }}$ parameter and the 6 other parameters, interactions which can therefore be assessed by the difference between $S T_{i}$ and $S_{i}$. The interaction terms of a set of parameters represent the fraction of $Y$ variance induced by the variance of these parameters but that cannot be explained by the sum of their main effects. The two sensitivity indices $S_{i}$ and $S T_{i}$ are equal if the effect of the $i^{\text {th }}$ parameter on the model output is independent of the values of the other parameters: in this case, there is no interaction between this parameter and the others and the model is said to be additive with respect to $\theta_{i}$.

\subsubsection{Extended FAST}

Sobol's method and Fourier Amplitude Sensitivity Test (FAST) are two of the most widely used methods to compute $S_{i}$ and $S T_{i}$ (Chan et al., 2000). We have chosen here to use the extended FAST (EFAST) method, which has been proved, in several studies (Saltelli and Bolado, 1998; Saltelli et al., 1999; Makowski et al., 2006), to be more efficient in terms of number of model evaluations than Sobol's method. The main difficulty in evaluating the first-order and total sensitivity indices is that they require the computation of high dimensional integrals. The EFAST algorithm performs a judicious deterministic sampling to explore the parameter space which makes it possible to reduce these integrals to onedimensional ones using Fourier decompositions. The reader interested in a detailed description of EFAST can refer to (Saltelli et al., 1999).

We have implemented the EFAST method in the Matlab® software, as well as a specific tool for computing and easily handling numerous STICS simulations. The uncertainties 
considered for the soil parameters are assumed independent and follow uniform distributions. These uncertainties are based to the measurements made in Chambry and correspond to the ranges of variation presented in Tab. 1. A preliminary study of the convergence of the sensitivity indices allowed us to set the number of simulations per parameter to 2000 , leading to a total number of model runs of $7 \times 2000=14000$ to compute the main and total effects for all output variables and parameters considered here. One run of the STICS model taking about 1s with a Pentium 4, $2.9 \mathrm{GHz}$ processor, the overall simulation process takes about $4 \mathrm{~h}$.

\subsubsection{Criteria based on GSA indices}

GSA provides main and total indices per parameter for each output variable considered. In order to summarize this information, we propose to create different criteria.

(i) The first one is a global measure of the information contained in a set of observations to estimate each parameter:

The Global Mean Sensitivity $\left(G M S_{i}\right)$ computes the mean of the main effect of parameter $\theta_{i}$ minus its interactions with the other parameters for all observed variables, each component being weighted by the degree of dependence of the corresponding output variable with the other variables:

$$
G M S_{i}=\frac{1}{K} \sum_{k=1}^{K}\left(1-\alpha_{k}\right)\left(S_{i}^{k}-R_{i}^{k}\right)
$$

where $k$ is a given observed output variable in a subset composed of $K$ variables among $\left\{L A I_{t}, Q N_{t}, t=1, \ldots, T\right.$ and $\left.Y l d\right\}, R_{i}^{k}=S T_{i}^{k}-S_{i}^{k}$ is the sum of all interaction terms including parameter $\theta_{i}$ for the observed variable $k, 0 \leq \alpha_{k} \leq 1$ is the mean of the absolute values of the correlation coefficients $\left|r_{k k^{\prime}}\right|$ between the variable $k$ and the other variables $k^{\prime}$ (calculated on the model simulations required for GSA): $\alpha_{k}=\frac{1}{K-1} \sum_{k^{\prime} \neq k}\left|r_{k k^{\prime}}\right|, K>1$.

The $G M S_{i}$ criterion is based on the following rules:

- if $S T_{i}$ is low (and thus $S_{i}$ ), observation $k$ is assumed not to contain enough information to estimate parameter $\theta_{i}$ : in this case the corresponding part of the criteria should be low,

- if $S_{i}$ is high (and thus $S T_{i}$ ), observation $k$ is assumed to contain sufficient information to estimate parameter $\theta_{i}$ : in this case the corresponding part of the criteria should be high,

- if $S_{i}$ is low and $S T_{i}$ is high, then the model is over-parameterized and difficulties in identifying parameter $\theta_{i}$ are expected (Ratto et al., 2007): in this case the corresponding part of the criteria should be low,

- high correlation between output variables indicates that the information contents of these variables are redundant: in this case the weights of the corresponding sensitivity indices should be reduced. 
$G M S_{i}$ varies within the range $[-1,1]$. It tends to 1 when $S_{i}$ is close to 1 for all observed variables and when all the observed variables are perfectly uncorrelated: in this case the model has an additive structure for the parameter $\theta_{i}$ and this parameter has a clearly identifiable influence on the K observed variables. $G M S_{i}$ tends to -1 when $S_{i}$ and $R_{i}^{k}$ are close to 0 and 1 respectively for all observed variables and when all the observed variables are perfectly uncorrelated: in this case problems of identification of the parameter $\theta_{i}$ are expected.

(ii) The second criterion is calculated at the whole parameter set level.

The Total Global Mean Sensitivity (TGMS), is the sum of the $G M S_{i}$ for all parameters:

$$
T G M S=\sum_{i=1}^{7} G M S_{i}=\sum_{i=1}^{7} \frac{1}{K} \sum_{k=1}^{K}\left(1-\alpha_{k}\right)\left(S_{i}^{k}-R_{i}^{k}\right)
$$

The TGMS criterion varies within the range $[-7,1]$. It tends to 1 when $R_{i}^{k}$ is close to 0 for all parameters and all observed variables and when all the observed variables are perfectly uncorrelated: in this case the model is additive. TGMS tends to -7 when $R_{i}^{k}$ is close to 1 for all parameters and all observed variables and when all the observed variables are perfectly uncorrelated: in this case the model is expected to be unidentifiable.

\subsection{Parameter estimation}

We chose a bayesian method which allow to take into account existing information on the parameters to be estimated (this improves the quality of the estimation process) and to compute an estimate of the posterior probability distribution of parameter values (Makowski et al., 2002; Gaucherel et al., 2008). More precisely, the method we chose is Importance Sampling.

\subsubsection{The Importance Sampling method}

The posterior parameter distribution is given by Bayes' theorem:

$$
\pi(\theta / Y)=\frac{\pi(Y / \theta) \pi(\theta)}{\pi(Z)}
$$

where $Y$ is the vector of total observations of size $K, \pi(\theta / Y)$ is the posterior parameter distribution, $\pi(\theta)$ is the prior parameter distribution, $\pi(Y)$ is a constant of proportionality determined by the requirement that the integral of $\pi(\theta / Y)$ over the parameter space equals 1 , and $\pi(Y / \theta)$ is the likelihood function. The likelihood is the probability of the data $Y$ given the parameters $\theta$. Its value is determined from the probability distribution of the errors of modelled and observed data. It is readily seen that both the prior distribution and the new data affect the posterior parameter distribution. 
The principle of the Importance Sampling method (Beven and Binley, 1992; Beven and Freer, 2001) is to approximate the posterior parameter distribution $\pi(\theta / Y)$ given in (7) by a discrete probability distribution $\left(\theta_{n}, p_{n}\right), n=1, \ldots, N, \sum_{n=1}^{N} p_{n}=1$, where $p_{n}$ is the probability associated with the parameter vector $\theta_{n}$. In our case, the method proceeds as follows:

(1) Randomly generate $N$ vectors $\theta_{n}, n=1, \ldots, N$, from the prior parameter distribution $\pi(\theta)$,

(2) Calculate the likelihood values $\pi\left(Y / \theta_{n}\right)$ for $n=1, \ldots, N$, associated with the different generated parameter vectors,

(3) Calculate $p_{n}=\frac{\pi\left(Y / \theta_{n}\right)}{\sum_{m=1}^{N} \pi\left(Y / \theta_{m}\right)}$

The pairs $\left(\theta_{n}, p_{n}\right), n=1, \ldots, N$, can be used to determine various characteristics of the posterior distribution, including the mean of the posterior joint distribution of $\theta$, $\bar{\theta}^{\text {post }}=\sum_{n=1}^{N} p_{n} \theta_{n}$.

In this study, we assume that the errors of simulated and observed data are independent between dates and variables and follow normal distributions of zero mean and standard deviation $\sigma_{k}$. Thus, we use the following likelihood function:

$$
\pi(Y / \theta)=\prod_{k=1}^{K} \frac{1}{\sqrt{2 \pi \sigma_{k}^{2}}} \exp \left\{-\frac{1}{2 \sigma_{k}^{2}}\left[y_{k}-f_{k}(\theta, x)\right]^{2}\right\}
$$

The parameters are assumed to be independent in our case. The prior distribution $\pi(\theta)$ is thus the product of the different marginal prior distributions. Accordingly to the distributions based on the experimental fields of Chambry we assumed them to be uniform and correspond to the uncertainties given in Tab. 1 .

We have implemented the Importance Sampling method in the Matlab® software. A preliminary study of the convergence of the estimates allowed us to set the total number of generated parameter vectors $N$ at 100000 .

\subsubsection{Criterion expressing the quality of parameter estimation}

(i) For each parameter, we created a criterion noted $R E_{i}$ (for Relative Error of the parameter $i)$, to quantify the quality of the parameter estimation. It computes the ratio between the error of the estimated parameter $\bar{\theta}_{i}{ }^{\text {post }}$ and the error of the prior information $\bar{\theta}_{i}{ }^{\text {prior }}$ : 


$$
R E_{i}=\frac{\operatorname{RMSE}\left(\bar{\theta}_{i}{ }^{\text {post }}\right)}{\operatorname{RMSE}\left({\overline{\theta_{i}}}^{\text {prior }}\right)}
$$

where $\operatorname{RMSE}\left(\bar{\theta}_{i}{ }^{\text {post }}\right)=\sqrt{\frac{1}{P} \sum_{p=1}^{P}\left(\theta_{i, p}^{\text {true }}-\bar{\theta}_{i, p}{ }^{\text {post }}\right)^{2}}, \theta_{i, p}^{\text {true }}$ is the true value of soil parameter $\theta_{i}$ for a given vector $p$ and $\bar{\theta}_{i, p}^{\text {post }}$ is the corresponding estimation given by the Bayesian method. $R E_{i}$ quantify how much the estimation given by the Bayesian method improves $\left(R E_{i}<1\right)$ or not $\left(R E_{i} \geq 1\right)$ the prior knowledge about the parameter value.

(ii) For all parameters, the criterion called Total Relative Error (TRE), is defined by the mean of the 7 values of $R E_{i}$ :

$$
\operatorname{TRE}=\frac{1}{7} \sum_{i=1}^{7} R E_{i}=\frac{1}{7} \sum_{i=1}^{7} \frac{\operatorname{RMSE}\left(\bar{\theta}_{i}^{\text {post }}\right)}{\operatorname{RMSE}\left(\bar{\theta}_{i}^{\text {prior }}\right)}
$$

\subsubsection{Criterion expressing the quality of prediction}

We created a criterion to quantify the quality of the prediction of the 3 agroenvironmental variables defined above. This criterion computes the ratio between the error of prediction obtained from the mean of the posterior distributions of the parameters, $\bar{\theta}^{\text {post }}$ and the one obtained from the mean of the prior distributions, $\bar{\theta}^{\text {prior }}$. It is called Relative Error of Prediction and is defined as follows:

$$
R E P_{j}=\frac{R M S E P_{j}\left(\bar{\theta}^{\text {post }}\right)}{R M S E P_{j}\left(\bar{\theta}^{\text {prior }}\right)}
$$

where $\operatorname{RMSEP}_{j}\left(\bar{\theta}^{\text {post }}\right)=\sqrt{\frac{1}{P \times Q_{j}} \sum_{p=1}^{P} \sum_{q=1}^{Q_{j}}\left(f_{j}^{q}\left(\theta_{p}^{\text {true }}\right)-f_{j}^{q}\left(\bar{\theta}_{p}^{\text {post }}\right)\right)^{2}}, \quad \theta_{p}^{\text {true }} \quad$ is the true values of soil parameters $\theta$ for a given vector $p, \bar{\theta}_{p}^{p o s t}$ is the corresponding estimation given by the Importance Sampling method, and $f_{j}^{q}\left(\theta_{p}^{\text {true }}\right)$ is assumed to be one of the $Q_{j}$ observations of the predicted variable $j$, for the $p^{\text {th }}$ vector of true values of soil parameters.

\subsection{Numerical experiments}

\subsubsection{Generation of observations for parameter estimation}

The STICS model output variables depend on the soil, climate and agronomic conditions for which the wheat crop is simulated. In view of this, we use different configurations in our study, as presented in Table 2: 4 contrasting climates, 2 different soil depths (shallow and deep), and 2 preceding crops (sugar beet and peas). The climatic data used were obtained from the meteorological station of Roupy $\left(49.48^{\circ} \mathrm{N}, 3.11^{\circ} \mathrm{E}\right)$. Four different sets of 
data were chosen to characterize a dry climate (1975-1976), a wet climate (1990-1991), a medium-dry climate (1979-1980) and a medium-wet climate (1972-1973). The distributions of soil parameters used in our study (GSA, creation of observations and prior information for GLUE) are independent and uniform and deduced from the experimental data acquired in Chambry (see Tab. 1). In this application, we assume that the type of soil depth (shallow or deep) and the preceding crop (sugar beet or pea) are known. As a consequence, two different ranges were considered for the depth of soil epc(2) and for the mineral nitrogen content at the beginning of the wheat crop simulation NO3init.

\begin{tabular}{|c|c|c|c|}
\hline $\begin{array}{l}\text { Climatic } \\
\text { conditions }\end{array}$ & $\begin{array}{l}\text { Soil } \\
\text { depth }\end{array}$ & $\begin{array}{l}\text { Preceding } \\
\text { crop }\end{array}$ & $\begin{array}{l}\text { Configuration } \\
\text { label }\end{array}$ \\
\hline Dry & Shallow & Sugar beet & dry-beet \\
\hline Medium-dry & Shallow & Sugar beet & mdry-beet \\
\hline Medium-wet & Shallow & Sugar beet & mwet-beet \\
\hline Wet & Shallow & Sugar beet & wet-beet \\
\hline Dry & Deep & Sugar beet & $d r y+b e e t$ \\
\hline Medium-dry & Deep & Sugar beet & $m d r y+b e e t$ \\
\hline Medium-wet & Deep & Sugar beet & mwet + beet \\
\hline Wet & Deep & Sugar beet & wet+beet \\
\hline Dry & Shallow & Pea & dry-pea \\
\hline Medium-dry & Shallow & Pea & mdry-pea \\
\hline Medium-wet & Shallow & Pea & mwet-pea \\
\hline Wet & Shallow & Pea & wet-pea \\
\hline Dry & Deep & Pea & $d r y+p e a$ \\
\hline Medium-dry & Deep & Pea & $m d r y+p e a$ \\
\hline Medium-wet & Deep & Pea & mwet+pea \\
\hline Wet & Deep & Pea & wet + pea \\
\hline
\end{tabular}

Table 2. Description of the 16 configurations based on soil, climatic and agronomic conditions.

We consider observations on wheat crops obtained for the different configurations described before. These observations consist of $L A I_{t}$ and $Q N_{t}$ available at 10 dates $t$, distributed through the wheat growing season: November 15, December 12, January 15, February 16, March 15, April 05, April 19, May 03, May 17 and June 07; and Yld. Three possible sets of observations (see Table 3 ) were considered for the parameter estimation experiments and the computation of the criteria based on the GSA and GLUE results. In order to compute observations, 50 vectors of true values $\theta^{\text {true }}$ were randomly generated from the distributions defined above. The number $P$ is thus equal to 50 . Corresponding values of STICS-wheat model output variables were simulated for each configuration leading to 50x16 simulations. Observations $y_{q, t}$ were then computed by adding a random error term to the simulated values of the variables and dates defined above:

$$
y_{q, t}=f_{q, t}\left(\theta^{\text {true }}, x\right)+\varepsilon_{q, t}
$$

where $f_{q, t}$ is the STICS model output $q\left(Y l d, L A I_{t}\right.$ or $\left.Q N_{t}\right)$ calculated on date $t$ (harvest for $Y l d$ or $t=1, \ldots, T$ for $L A I$ and $Q N), x$ is the vector of explanatory variables and $\varepsilon_{q, t}$ is the 
observation error term. Following the assumptions made in Section 2.3.1 to compute the likelihood function of the GLUE method, the vector of observation error is given by: $\varepsilon_{q, t} \sim N\left(0, \sigma_{q, t}^{2}\right)$ where $\sigma_{q, t}=\sigma_{q}^{0} f_{q, t}\left(\theta^{\text {true }}, x\right), \quad \sigma_{Y l d}^{0}=9 \%, \sigma_{L A I}^{0}=17 \%$ and $\sigma_{Q N}^{0}=30 \%$ according to measurements realized in agricultural plots (Machet et al., 2007; Moulin et al., 2007).

\begin{tabular}{lll}
\hline Set number & Variables used & Size $K$ \\
\hline 1 & $L A I_{t}$ on dates $t=1, \ldots, 10$ & $K=10$ \\
2 & $L A I_{t}$ and $Q N_{t}$ on dates $t=1, \ldots, 10$ & $K=20$ \\
3 & $L A I_{t}$ and $Q N_{t}$ on dates $t=1, \ldots, 10$, and $Y l d$ & $K=21$ \\
\hline
\end{tabular}

Table 3. Description of the 3 sets of observations.

\subsubsection{Generation of observations for prediction}

The prediction of variables of interest is performed on independent wheat crop seasons from those used in the estimation process. From each of the 50 vectors defined above, 120 configurations of prediction were studied and are composed by the corresponding type of soil depth, 10 climatic data, 3 different sowing dates and 4 different cropping techniques (2 amount of fertiliser and 2 types of preceding crops). The number $Q_{j}$ is thus equal to 120 The climatic data were obtained from the meteorological station of Roupy $\left(49.48^{\circ} \mathrm{N}, 3.11^{\circ} \mathrm{E}\right)$ and are different from those used in the parameter estimation process.

From each vector $\theta^{\text {true }}$, type of soil depth and configuration, the values of synthetic observations of the predicted variables of interest (Yld, Prot and Nit at harvest) are simulated with STICS-wheat. The values of the permanent properties (argi, Norg, epc(2), HCC(1) and $H C C(2))$ of $\theta^{\text {true }}$ are the same as those used to create the synthetic observations in the estimation step and the initial conditions (Hinit and NO3init) are randomly generated from the distributions defined in Tab. 1: we assume that the values of the initial conditions are not known for the predicted season. For each parameter estimation experiment defined above, each estimated values of the permanent properties are used to predict the output variables of wheat crop through the STICS model. Assuming that the initial conditions are unknown for the predicted season, they are fixed at the mean of their distribution.

\section{Results}

\subsection{Relationship between criteria based on GSA results and the quality of estimates}

\subsubsection{At a single parameter level}

For each soil parameter, we present here the results about the relationship between the criterion based on GSA results and the criterion related to the quality of the parameter estimate, for the three sets of observations and the 16 soil, climatic and agronomic conditions.

Figure 1 shows that a good link exists between $G M S_{i}$ and $R E_{i}$ : the relationship seems to be linear, the higher the $G M S_{i}$ criterion, the lower the $R E_{i}$ and the better the quality of estimation of the $i^{\text {th }}$ parameter. The results show clusters of parameters: $h$ (Hinit) at high $G M S_{i}$ and low $R E_{i}$ values, $e(\operatorname{epc}(2))$ at intermediate $G M S_{i}$ and $R E_{i}$ values, and the other parameters all grouped in the same cluster at low $G M S_{i}$ and high $R E_{i}$ values. Within the 
scattering around the relationship, the position of the parameter depends on the configuration and especially the soil depth.

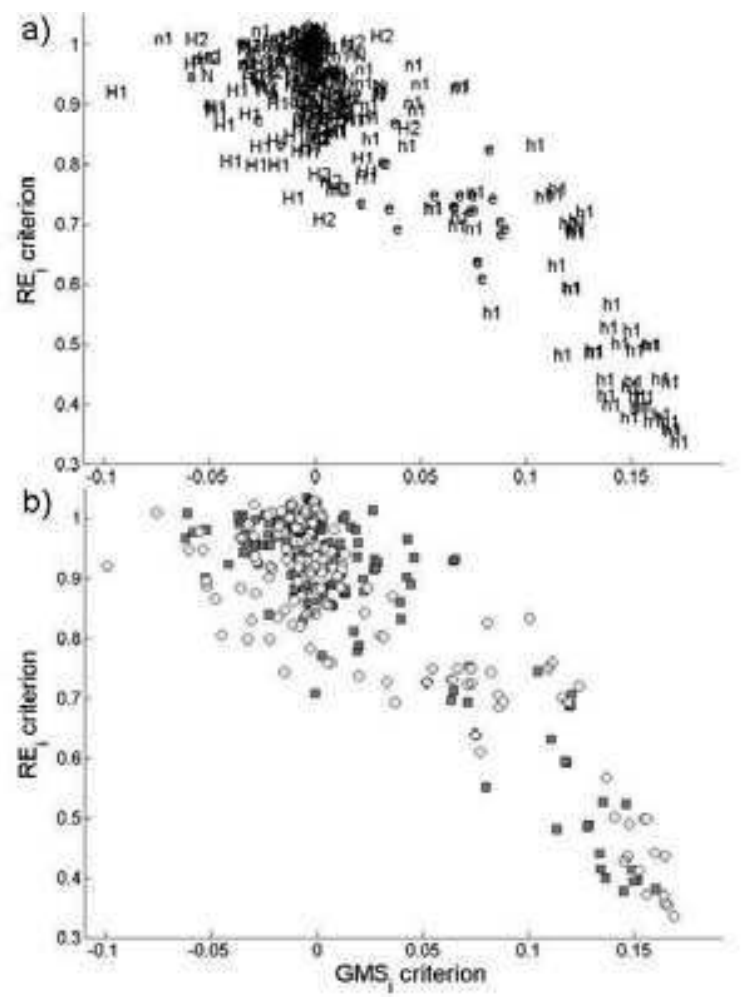

Fig. 1. Scatter diagrams of the criteria $R E_{i}$ and $G M S_{i}$ of the 7 soil parameters for the 3 sets of observations and the 16 configurations. a) Label a corresponds to argi, $N$ to Norg, $e$ to $\operatorname{epc}(2), H 1$ to HCC(1), H2 to HCC(2), $h$ to Hinit and $n$ to NO3init. b) Symbol o corresponds to shallow soils and $\square$ corresponds to deep soils.

The case of parameters for which the observations contain enough information to estimate them precisely can be illustrated by the parameter Hinit. For example, for the dry-beet configuration and the observation set \#1, Hinit has a big main effect (equal to 0.582 on average), leading to a high value of $G M S_{i}=0.155$. In this case, the parameter Hinit has a low value of $R E_{i}\left(R E_{i}=0.499\right)$ meaning a good improvement of its uncertainty through the parameter estimation process (see Figure 1a). In general, the results show that for high values of $G M S_{i}$, the reduction of the estimation error is large: a high GMS $i$ indicates good parameter estimation.

Small first-order indices combined with a large interaction are thought to induce problems of identification. In our case, this applies to most of the parameters. For example, $H C C(1)$ has a small main effect ( 0.089 on average) and a large interaction (equal to 0.251 on average) for the wet-beet configuration and the first observation set, leading to a negative value of $G M S_{i}=-0.099$. In this case, the parameter $H C C(1)$ has a high value of 
$R E_{i}\left(R E_{i}=0.921\right)$, meaning a poor improvement in parameter estimation (see Figure 1a). In general, the results show that for negative values of $G M S_{i}$, the reduction of the estimation error is small: a negative value of $G M S_{i}$ reveals a bad quality of the parameter estimation. The values of both criteria for parameter epc(2) vary a lot between the different configurations and especially between the types of soil depth. As it is shown is Figure 1b, only configurations with a shallow soil (with the symbolo) allow retrieving the parameter $\operatorname{epc}(2)$. For shallow soil the observed variables are quite sensitive to epc(2), leading to a quite good estimate, while for deep soil the observed variables are no longer sensitive to epc(2), leading to a poor estimate. For example, the configuration dry-beet and the set \#3 leads to intermediate $G M S_{i}$ and $R E_{i}$ values $\left(G M S_{i}=0.073\right.$ and $\left.R E_{i}=0.724\right)$, while dry+beet and set \#3 leads to lower (resp. higher) $G M S_{i}$ (resp. $\left.R E_{i}\right)$ values $\left(G M S_{i}=-0.008\right.$ and $\left.R E_{i}=0.871\right)$.

In order to quantify the quality of the relationship illustrated in Figure 1, we propose to compute the Spearman rank correlations coefficient (Spearman, 1904) between $G M S_{i}$ and $R E_{i}$, for each configuration and observation set. This coefficient allows comparing the relationship between two ranking lists. The analysis is made after discarding the parameters having a negative $G M S_{i}$ which have always a poor quality of estimation and whose rank would still be high. The results displayed in Table 4 show that the averaged Spearman's correlation between $G M S_{i}$ and $R E_{i}$ is satisfactory (about $75.4 \%$ ). The $G M S_{i}$ criterion is thus considered to be effective for ranking the accessible parameters (for which the criterion is positive) with respect to their quality of estimates.

\begin{tabular}{|c|c|c|c|c|}
\hline & Parameters* & & Climates & Observation sets \\
\hline$\left(R E_{i}, G M S_{i}\right)$ & $75 \%$ & (TRE, TGMS) & $72 \%$ & $91 \%$ \\
\hline
\end{tabular}

Table 4. Averaged Spearman's rank correlation coefficient of criteria $\left(R E_{i}, G M S_{i}\right)$ and (TRE, TGMS). The first pair of criteria is involved in the parameter ranking and the second pair is involved in the climates and observation sets ranking.

\subsubsection{At the whole parameter set level}

In Figure 2 the values of TRE and TGMS have been averaged for each observation set and climate over the two soil depths and the two preceding crops. The relationship between TRE and TGMS appears satisfactory. The TRE criterion never reaches low values (the minimum value is about 0.8 ) even for high TGMS values (about 0.21 ), due to the relatively large number of parameters which are not easily retrievable. The effect of climate is striking. Configurations with a dry climate have the higher values of TGMS (between 0.16 and 0.2 ) and they correspond to the best quality of estimation of the parameter set (TRE between 0.81 and 0.86 ), unlike configurations with a wet climate (TGMS below 0.03 and TRE above 0.89).

The greater the number of observations considered in the estimation process (from set \#1 to set \#3), the lower is the TRE. As expected and seen in Figure 2, TGMS often decreases when the number of observations increases. Although some of the observed variables are mutually correlated (the average correlation coefficient between set \#1 and set \#2 is about $61 \%$ while it is about $37 \%$ between set \#2 and set \# 3), they each improve the quality of the parameter set estimation. 
Finally, the Spearman's correlation coefficients between TGMS and TRE were computed for each type of soil depth, preceding crop and observation set, in order to quantify the ranking of the 4 climates given by both TGMS and TRE. The averaged Spearman's correlation presented in Table 4 between TGMS and TRE is satisfactory (about $72 \%$ ). Secondly, the Spearman correlations between TGMS and TRE were computed for each soil depth, preceding crop and climate, in order to quantify the ranking of the three observation sets given by both TGMS and TRE. The averaged Spearman's correlation between TGMS and TRE is very satisfactory (about $91 \%$ ).

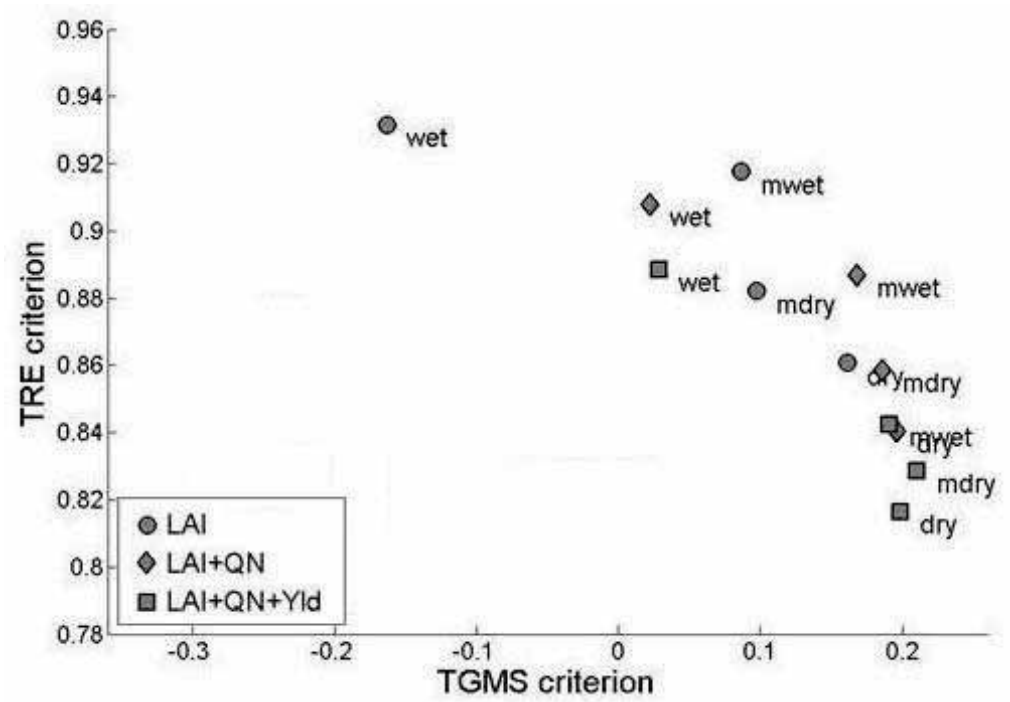

Fig. 2. Scatter diagrams of the criteria TRE and TGMS at the whole parameter set level, for the 3 sets of observations and the 4 types of climate. Each of the 12 points is an average of the 2 soil depth and the 2 preceding crop configurations. The symbol $\circ$ corresponds to the set \#1, $\diamond$ to set \#2 and $\boldsymbol{m}$ to set \#3.

This study shows that the quality of parameter estimation can be explained by the results of GSA. Suitable empirical criteria have been proposed to summarize the results of GSA which allow ranking (i) the parameters with respect to their quality of estimate and (ii) the configurations (particularly the climate and the observation set) with respect to the quality of estimation of the whole parameter set. These criteria are thus shown in our case to be useful tools for estimating the potential of given configurations of observations for retrieving soil parameter values. They may be used also for optimizing the type of observations to be acquired and the dates of acquisition.

\subsection{Impact of the quality of estimates on the quality of prediction}

The quality of the prediction in now analysed. Figure 3 and 4 shows the results in term of $R E P_{j}$ for the prediction of the variables of interest concerning wheat crop, by using the estimated values of the permanent soil parameters (argi, Norg, epc(2), HCC(1) and HCC(2)) in place of their prior values. The initial conditions (Hinit and NO3init) are assumed to be 
unknown for the prediction and are fixed at the mean of their distributions. The values of $R E P_{j}$ are calculated for the 2 types of soil depth and the 3 sets of observations.

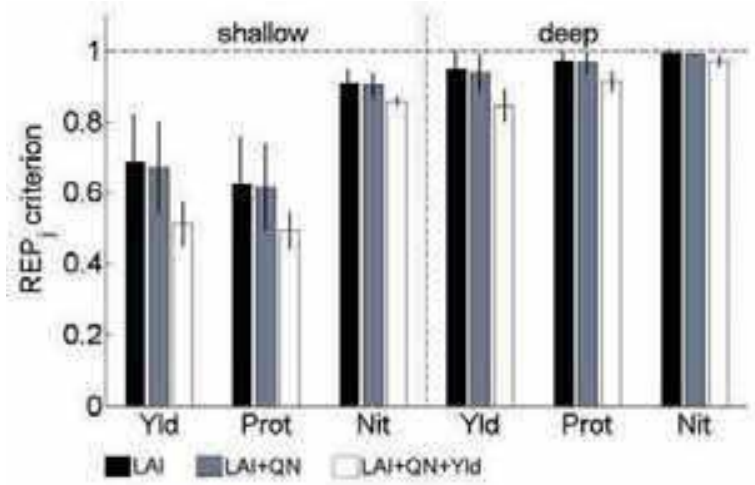

Fig. 3. Results of the prediction of the variables of interest. The results are presented for the 2 types of soil depth and the 3 sets of observations and averaged on the 4 climates.

In Figure $3, R E P_{j}$ values are averaged on the 4 climates. It can be seen that 2 of the 3 variables of interest ( $Y l d$ and Prot) have a significant lower $R E P_{j}$ and therefore a greatly improved quality of prediction when using the estimated values of the permanent parameters, as compared to when using prior information on the parameters. In that case, Yld and Prot seem to be quite sensitive to the permanent soil parameters. The output variable Nit is not or slightly affected by the estimation of the soil parameters because it is sensitive to the initial conditions, which are fixed at a nominal value for the prediction, and not to the permanent parameters. Through the estimation of the permanent soil properties, the size of the observation set slightly improves the quality of prediction: the bigger the observation set size the better the permanent parameter estimates and the better the prediction. The most important improvement between two sets of observations concerns the output variable $Y l d$ in a shallow soil: $R E P_{j}$ is about 0.66 for set \#2 and about 0.52 for set \#3. In that case, a lot of information is provided by the observation of Yld.

The type of soil depth affects a lot the quality of the prediction and especially for the output variables Yld and Prot, which have a lower $R E P_{j}$ when the type of soil is shallow. It is not surprising, accordingly to the results of parameter estimation, because the parameter epc(2) has a better quality of estimates in the case of shallow soil and because Yld and Prot are also quite sensitive to this parameter, as well as the observed variables (see Section 3.1.1). The output variable Nit is not affected by the soil depth because of its lack of sensitivity to epc(2). 


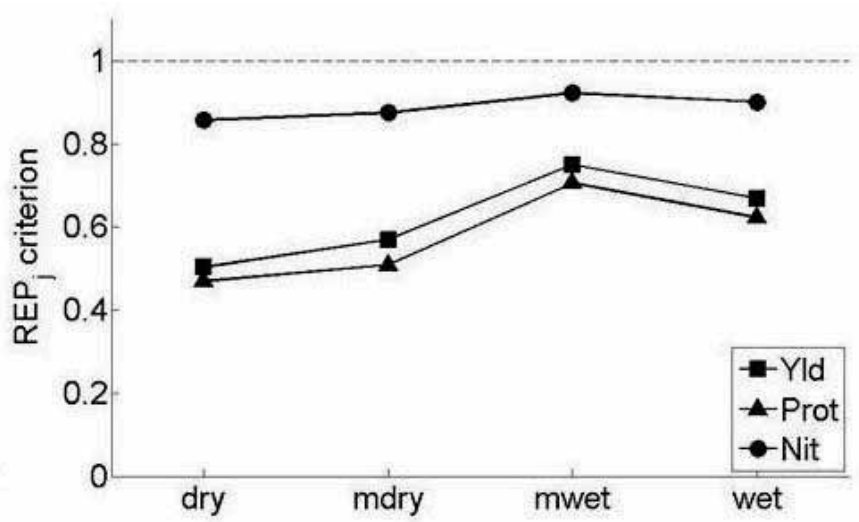

Fig. 4. Results of the prediction of the variables of interest. Effect of the climates on prediction of the variables of interest. The results are averaged on the 3 observation sets for a shallow soil.

The effect of climate on the quality of prediction of the variables of interest is illustrated in Figure 4 on a shallow soil and averaged over the 3 sets of observations. The dryer the climate the better the quality of the prediction. As the observed variables are more sensitive to the soil parameters in dry climatic conditions (see Section 3.1.2), that allows a better quality of the parameter estimates in that case. The results of the prediction are thus affected by the quality of the estimates and are better in dry climatic conditions, because of their sensitivity on the parameters. The $R E P_{j}$ value of the output variables Yld and Prot significantly decreases from 0.71 to 0.5 and 0.65 to 0.47 . As previously, the output variable $N i t$ is not accurately predicted and slightly affected by the climate: $R E P_{j}$ decreases from 0.92 to 0.86 .

It was shown in this study that some soil parameters can be retrieved by considering observations on crops and that the estimated values of the permanent soil parameters allow reducing the uncertainty on the prediction, because of the sensitivity of the predicted variables on these parameters. The quality of the prediction is manly affected by two factors: the soil depth and climate. These results are closely linked to the quality of the parameter estimates.

\section{Conclusion}

In our results the link between the quality of parameter estimation and GSA results was illustrated through three types of behavior: high first-order indices are associated with good quality of estimation, low total indices are associated with bad quality of estimation, and high total indices combined with low first-order indices are associated with poor estimates because of interactions between parameters. Many other studies show that the parameter estimation performance can be explained by the results of GSA (Tremblay and Wallach, 2004; Gaucherel et al., 2008; Manache and Melching, 2008). Given the large number of output variables and dates considered in this application, the GSA indices had to be summarized to study the link between GSA and parameter estimation results. We 
proposed the $G M S_{i}$ criterion and show its relation with the criterion $R E_{i}$ which measures the quality of estimation of parameter $i$. The criterion $G M S_{i}$ proves to be effective for ranking the accessible parameters with respect to their quality of estimation. For a given configuration, GSA is able to provide information on which parameters can be estimated and which can be fixed as they do not deserve an accurate determination (Ratto et al., 2007). We show in this work that the parameters having $G M S_{i}$ close to zero are not accessible from the observations and the STICS model.

The total criterion TGMS can be used to predict the ranking of the configurations with respect to their ability to retrieve the whole set of parameters, and in particular the ranking of the climates and the observation sets: it is possible to predict which type of climate and observation set will lead to the better estimation of the whole parameter set. These results are particularly interesting for screening the possibility of estimating parameters from a given set of available observations in a given agro-environmental context, and, following Kontoravdi et al. (2005), promote GSA as an excellent precursor to optimal experimental design.

From observations on crop status, it is possible to retrieve the soil parameters and the estimated values allow improving the quality of the prediction of agro-environmental variables. Among them, some variables are strongly affected by the quality of the parameter estimates, such as grain yield and protein of the grain, because of their large sensitivity on the permanent soil parameters. This result is particularly interesting for agro-environmental work because the criteria based on GSA also allow screening the possibility of a given set of available observations to predict, through soil parameter estimates, the variables of interest for crop management.

Finally, it would be helpful to conduct such a study on real data to assess the impact of model errors on both soil parameter retrieval and link between the proposed criteria.

\section{Acknowledgements}

The financial support provided by CNES and Arvalis-Institut du Végétal is gratefully acknowledged.

\section{References}

Baret, F., Houles, V.,Guerif, M., 2006. Quantification of plant stress using remote sensing observations and crop models: the case of nitrogen management. Symposium on Imaging Techniques for Understanding Plant Reponses to Stress held at the Society-for-Experimentaal-Biology Meeting. Canerbury, ENGLAND.

Batchelor, W.D., Basso, B.,Paz, J.O., 2002. Examples of strategies to analyze spatial and temporal yield variability using crop models. European Journal of Agronomy 18 141-158.

Beven, K.,Binley, A., 1992. The Future of Distributed Models - Model Calibration and Uncertainty Prediction. Hydrological Processes 6 279-298.

Beven, K.,Freer, J., 2001. Equifinality, data assimilation, and uncertainty estimation in mechanistic modelling of complex environmental systems using the GLUE methodology. Journal of Hydrology 249 11-29. 
Blackmore, S.,Moore, M., 1999. Remedial correction of yield map data. Precision Agriculture 1 53-56.

Brisson, N., Ruget, F., Gate, P., Lorgeou, J., Nicoullaud, B., Tayot, X., Plenet, D., Jeuffroy, M.H., Bouthier, A., Ripoche, D., Mary, B.Juste, E., 2002. STICS: a generic model for simulating crops and their water and nitrogen balances. II. Model validation for wheat and maize. Agronomie 22 69-92.

Campolongo, F.,Saltelli, A., 1997. Sensitivity analysis of an environmental model an application of different analysis methods. Reliability Engineering \& System Safety 57 49-69.

Chan, K., Tarantola, S., Saltelli, A.,Sobol, I.M., 2000. Variance-based methods. In: Saltelli, A., Chan, K.,Scott, E.M., (Eds.), Sensitivity analysis. Wiley: New York.

Ferreyra, R.A., Jones, J.W.,Graham, W.D., 2006. Parameterizing Spatial Crop Models with Inverse Modeling: Sources of Error and Unexpected Results. Transactions of the ASABE 49 1547-1561.

Gabrielle, B., Roche, R., Angas, P., Cantero-Martinez, C., Cosentino, L., Mantineo, M., Langensiepen, M., Henault, C., Laville, P., Nicoullaud, B.,Gosse, G., 2002. A priori parameterisation of the CERES soil-crop models and tests against several European data sets. Agronomie 22 119-132.

Gaucherel, C., Campillo, F., Misson, L., Guiot, J.,Boreux, J.-J., 2008. Parameterization of a process-based tree-growth model: Comparison of optimization, MCMC and Particle Filering algorithms. Environmental Modelling \& Software 23 1280-1288.

Golovko, L.,Pozdnyakov, A.I., 2007. Electrical geophysical methods in agriculture. Progress of Information Technology in Agriculture 457-471.

Gomez-Delgado, M.,Tarantola, S., 2006. GLOBAL sensitivity analysis, GIS and multicriteria evaluation for a sustainable planning of a hazardous waste disposal site in Spain. International Journal of Geographical Information Science 20 449-466.

Guérif, M., Beaudoin, N., Durr, C., Machet, J.M., Mary, B., Michot, D., Moulin, D., Nicoullaud, B.,Richard, G., 2001. Designing a field experiment for assessing soil and crop spatial variability and defining site specific management strategies. Proceedings 3rd European Conference on Precision Agriculture. Montpellier, France.

Hadria, R., Khabba, S., Lahrouni, A., Duchemin, B., Chehbouni, A., Carriou, J.,Ouzine, L., 2007. Calibration and validation of the STICS crop model for managing wheat irrigation in the semi-arid Marrakech/Al Haouzi plain. Arabian Journal for Science and Engineering 32 87-101.

Houborg, R.,Boegh, E., 2008. Mapping leaf chlorophyll and leaf area index using inverse and forward canopy reflectance modeling and SPOT reflectance data. Remote Sensing of Environment 112 186-202.

Houlès, V., Mary, B., Guérif, M., Makowski, D.,Juste, E., 2004. Evaluation of the crop model STICS to recommend nitrogen fertilization rates according to agroenvironmental criteria. Agronomie 24 1-9.

Irmak, A., Jones, J.W., Batchelor, W.D.,Paz, J.O., 2001. Estimating Spatially Variable Soil Properties for Application of Crop Models in Precision Farming. Transactions of the ASAE 44 1343-1353.

King, D., Daroussin, J.,Tavernier, R., 1994. Development of a Soil Geographic Database from the Soil Map of the European Communities. Catena 21 37-56. 
Kontoravdi, C., Asprey, S.P., Pistikopoulos, E.N.,Mantalaris, A., 2005. Application of global sensitivity analysis to determine goals for design of experiments: An example study on antibody-producing cell cultures. Biotechnology Progress 21 1128-1135.

Lagacherie, P., Baret, F., Feret, J.B., Netto, J.M.,Robbez-Masson, J.M., 2008. Estimation of soil clay and calcium carbonate using laboratory, field and airborne hyperspectral measurements. Remote Sensing of Environment 112 825-835.

Launay, M.,Guérif, M., 2003. Ability for a model to predict crop production variability at the regional scale: an evaluation for sugar beet. Agronomie 23 135-146.

Launay, M.,Guérif, M., 2005. Assimilating remote sensing data into a crop model to improve predictive performance for spatial applications. Agriculture Ecosystems \& Environment 111 321-339.

Machet, J.M., Couturier, A.,Beaudoin, N., 2007. Cartographie du rendement du blé et des caractéristiques qualitatives des grains. In: Guérif, M.,King, D., (Eds.), Agriculture de précision. Quae: Versailles.

Makowski, D., Naud, C., Jeuffroy, M.H., Barbottin, A.,Monod, H., 2006. Global sensitivity analysis for calculating the contribution of genetic parameters to the variance of crop model prediction. Reliability Engineering \& System Safety 91 1142-1147.

Makowski, D., Wallach, D.,Tremblay, M., 2002. Using a Bayesian approach to parameter estimation; comparison of the GLUE and MCMC methods. Agronomie 22 191203.

Manache, G.,Melching, C.S., 2008. Identification of reliable regression- and correlationbased sensitivity measures for importance ranking of water-quality model parameters. Environmental Modelling \& Software 23 549-562.

Moulin, S., Zurita, R.M.,Guérif, M., 2007. Estimation de variables biophysiques du couvert par ajustement de modèles de transfert radiatif sur des réflectances. In: Guérif, M.,King, D., (Eds.), Agriculture de précision. Quae: Versailles.

Murphy, B., Geeves, G., Miller, M., Summerell, G., Southwell, P.,Rankin, M., 2003. The application of pedotransfer functions with existing soil maps to predict soil hydraulic properties for catchment-scale hydrologic and salinity modelling. International Congress on Modelling and Simulation. Townsville, AUSTRALIA.

Pierce, F.J., Nowak, P.,Roberts, P.C., 1999. Aspects of Precision Agriculture. Advances in Agronomy 67 1-85.

Ratto, M., Young, P.C., Romanowicz, R., Pappenberger, F., Saltelli, A.,Pagano, A., 2007. Uncertainty, sensitivity analysis and the role of data based mechanistic modeling in hydrology. Hydrology and Earth System Sciences 11 1249-1266.

Reynolds, C.A., Jackson, T.J.,Rawls, W.J., 2000. Estimating soil water-holding capacities by linking the Food and Agriculture Organization soil map of the world with global pedon databases and continuous pedotransfer functions. Water Resources Research 36 3653-3662.

Ruget, F., Brisson, N., Delecolle, R.,Faivre, R., 2002. Sensitivity analysis of a crop simulation model, STICS, in order to choose the main parameters to be estimated. Agronomie 22 133-158.

Saltelli, A.,Bolado, R., 1998. An alternative way to compute Fourier amplitude sensitivity test (FAST). Computational Statistics \& Data Analysis 26 445-460. 
Saltelli, A., Tarantola, S.,Campolongo, F., 2000. Sensitivity analysis as an ingredient of modeling. Statistical Science 15 377-395.

Saltelli, A., Tarantola, S.,Chan, K.P.S., 1999. A quantitative model-independent method for global sensitivity analysis of model output. Technometrics 41 39-56.

Spearman, C., 1904. The proof and measurement of association between two things. Amer. J. Psychol. 15 72-101.

Tremblay, M.,Wallach, D., 2004. Comparison of parameter estimation methods for crop models. Agronomie 24 351-365.

Weiss, M.,Baret, F., 1999. Evaluation of canopy biophysical variable retrieval performances from the accumulation of large swath satellite data. Remote Sensing of Environment 70 293-306. 
Advances in Geoscience and Remole Sensing

Advances in Geoscience and Remote Sensing

Edited by Gary Jedlovec

ISBN 978-953-307-005-6

Hard cover, 742 pages

Publisher InTech

Published online 01, October, 2009

Published in print edition October, 2009

Remote sensing is the acquisition of information of an object or phenomenon, by the use of either recording or real-time sensing device(s), that is not in physical or intimate contact with the object (such as by way of aircraft, spacecraft, satellite, buoy, or ship). In practice, remote sensing is the stand-off collection through the use of a variety of devices for gathering information on a given object or area. Human existence is dependent on our ability to understand, utilize, manage and maintain the environment we live in - Geoscience is the science that seeks to achieve these goals. This book is a collection of contributions from world-class scientists, engineers and educators engaged in the fields of geoscience and remote sensing.

\section{How to reference}

In order to correctly reference this scholarly work, feel free to copy and paste the following:

Hubert Varella, Martine Guerif and Samuel Buis (2009). Estimation of Soil Properties Using Observations and the Crop Model STICS. Interest of Global Sensitivity Analysis and Impact on the Prediction of AgroEnvironmental Variables, Advances in Geoscience and Remote Sensing, Gary Jedlovec (Ed.), ISBN: 978-953307-005-6, InTech, Available from: http://www.intechopen.com/books/advances-in-geoscience-and-remotesensing/estimation-of-soil-properties-using-observations-and-the-crop-model-stics-interest-of-global-sensiti

\section{INTECH}

open science | open minds

\section{InTech Europe}

University Campus STeP Ri

Slavka Krautzeka 83/A

51000 Rijeka, Croatia

Phone: +385 (51) 770447

Fax: +385 (51) 686166

www.intechopen.com

\section{InTech China}

Unit 405, Office Block, Hotel Equatorial Shanghai

No.65, Yan An Road (West), Shanghai, 200040, China 中国上海市延安西路65号上海国际贵都大饭店办公楼 405 单元 Phone: +86-21-62489820

Fax: +86-21-62489821 
(C) 2009 The Author(s). Licensee IntechOpen. This chapter is distributed under the terms of the Creative Commons Attribution-NonCommercial-ShareAlike-3.0 License, which permits use, distribution and reproduction for non-commercial purposes, provided the original is properly cited and derivative works building on this content are distributed under the same license. 